Pacific Journal of Mathematics

DISCRETE GENERALIZED GRONWALL INEQUALITIES IN 


\title{
DISCRETE GENERALIZED GRONWALL INEQUALITIES IN THREE INDEPENDENT VARIABLES
}

\author{
B. G. Pachpatte and S. M. Singare
}

The objective of this paper is to establish some new discrete inequalities of the Gronwall type in three independent variables which can be used in the analysis of a class of finite difterence equations involving three independent variables.

1. Introduction. The role played by the discrete inequalities of the Gronwall type [3] in the theory of finite difference equations and numerical analysis is well known (see, [4]-[8] and the references therein). Recently, in a series of papers [4]-[8], Pachpatte has established a number of new discrete inequalities of the Gronwall type which can be used in the theory of discrete time systems involving one independent variable. To our knowledge such inequalities have not been considered before and seem to have much future in the literature.

2. Main results. Before giving the main results in this section, we first recollect a few of the basic notions and definitions from [4]-[8]. Let $N_{0}=\{0,1,2, \cdots\}$. The expression $u(0)+\sum_{s=0}^{n-1} b(s)$ represents a solution of the linear difference equation $\Delta u(n)=b(n)$ for all $n \in N_{0}$, where $\Delta$ is the operator defined by $\Delta u(n)=u(n+1)-$ $u(n)$. The expression $u(0) \prod_{s=0}^{n-1} c(s)$ represents a solution of the linear difference equation $u(n+1)=c(n) u(n)$ for all $n \in N_{0}$. We use the usual convention of writing $\sum_{s \in \Phi} b(s)=0$ and $\Pi_{s \in \Phi} c(s)=1$, if $\Phi$ is the empty set. We also use the following notions of the operators $\Delta u_{x}(x, y, z)=u(x+1, y, z)-u(x, y, z), \quad \Delta u_{y}(x, y, z)=u(x, y+1, z)-$ $u(x, y, z), \Delta u_{z}(x, y, z)=u(x, y, z+1)-u(x, y, z)$ and $\Delta u_{x y}^{2}(x, y, z)=$ $\Delta u_{x}(x, y+1, z)-\Delta u_{x}(x, y, z)$ and so on. We often use the letters $x$, $y$, and $z$ to denote the three independent variables which are the members of $N_{0}$. For $x, y, z \in N_{0}$, and functions $a, b, c$ with domain $N_{0}$, and $p$ with domain $N_{0}^{3}$, set

$$
\begin{aligned}
\phi(x, y, z ; a, b, c ; p)= & {[a(0)+b(y)+c(z)] \prod_{s=0}^{x-1}\left[1+\frac{\Delta a(s)}{a(s)+b(0)+c(z)}\right.} \\
& \left.+\sum_{t=0}^{y-1} \sum_{r=0}^{z-1} p(s, t, r)\right] .
\end{aligned}
$$

A useful three independent variable discrete inequality is embodied in the following theorem. 
THEOREM 1. Let $u(x, y, z)$ and $p(x, y, z)$ be real-valued nonnegative functions defined for $(x, y, z) \in N_{0}^{3}$ for which the inequality

$$
u(x, y, z) \leqq a(x)+b(y)+c(z)+\sum_{s=0}^{x-1} \sum_{t=0}^{y-1} \sum_{r=0}^{z-1} p(s, t, r) u(s, t, r),
$$

holds for $(x, y, z) \in N_{0}^{3}$, where $a(x), b(y), c(z)>0 ; \Delta a(x), \Delta b(y), \Delta c(z)>0$ are real-valued functions defined on $N_{0}$. Then

$$
u(x, y, z) \leqq \phi(x, y, z ; a, b, c ; p)
$$

for $(x, y, z) \in N_{0}^{3}$.

Proof. Define a function $m(x, y, z)$ by

$$
m(x, y, z)=a(x)+b(y)+c(z)+\sum_{s=0}^{x-1} \sum_{t=0}^{y-1} \sum_{r=0}^{z-1} p(s, t, r) u(s, t, r),
$$

so that, by definition

$$
\begin{aligned}
& m(0, y, z)=a(0)+b(y)+c(z), \\
& m(x, 0, z)=a(x)+b(0)+c(z), \\
& m(x, y, 0)=a(x)+b(y)+c(0) .
\end{aligned}
$$

Then

$$
\Delta m_{x}(x, y, z)=\Delta a(x)+\sum_{t=0}^{y-1} \sum_{r=0}^{z-1} p\left(x, t, r^{r}\right) u(x, t, r),
$$

and from (3) we have

$$
\begin{aligned}
& \Delta m_{x}(x, y+1, z)-\Delta m_{x}(x, y, z)=\sum_{r=0}^{z-1} p(x, y, r) u(x, y, r), \\
& \Delta m_{x}(x, y+1, z+1)-\Delta m_{x}(x, y, z+1)=\sum_{r=0}^{z} p(x, y, r) u(x, y, r) .
\end{aligned}
$$

From (4) and (5) we have

$$
\Delta^{2} m_{x y}(x, y, z+1)-\Delta^{2} m_{x y}(x, y, z)=p(x, y, z) u(x, y, z),
$$

which in view of (1) implies

$$
\Delta^{2} m_{x y}(x, y, z+1)-\Delta^{2} m_{x y}(x, y, z) \leqq p(x, y, z) m(x, y, z) .
$$

From the definition of $m(x, y, z)$ we observe that $m(x, y . z)^{9} \leqq$ $m(x, y, z+1)$, for $(x, y, z) \in N_{0}^{3}$. Using this fact in (6) we have

$$
\Delta^{2} m_{x y}(x, y, z+1)-\Delta^{2} m_{x y}(x, y, z) \leqq p(x, y, z) m(x, y, z+1),
$$




$$
\frac{\Delta^{2} m_{x y}(x, y, z+1)}{m(x, y, z+1)}-\frac{\Delta^{2} m_{x y}(x, y, z)}{m(x, y, z+1)} \leqq p(x, y, z) .
$$

From (7) we observe that

$$
\frac{\Delta^{2} m_{x y}(x, y, z+1)}{m(x, y, z+1)}-\frac{\Delta^{2} m_{x y}(x, y, z)}{m(x, y, z)} \leqq p(x, y, z) .
$$

Now keeping $x, y$ fixed in (8), set $z=r$ and sum over $r=0,1 \cdots$, $z-1$ to obtain the estimate

$$
\frac{\Delta^{2} m_{x y}(x, y, z)}{m(x, y, z)} \leqq \sum_{r=0}^{z-1} p(x, y, r) \text {. }
$$

From (9) and in view of the fact that $m(x, y, z) \leqq m(x, y+1, z)$ we observe that

$$
\frac{\Delta m_{x}(x, y+1, z)}{m(x, y+1, z)}-\frac{\Delta m_{x}(x, y, z)}{m(x, y, z)} \leqq \sum_{r=0}^{z-1} p(x, y, r) .
$$

Keeping $x, z$ fixed in (10), set $y=t$ and sum over $t=0,1, \cdots, y-1$ to obtain the estimate

$$
\begin{aligned}
m(x & +1, y, z) \\
& \leqq m(x, y, z)\left[1+\frac{\Delta a(x)}{a(x)+b(0)+c(z)}+\sum_{t=0}^{y-1} \sum_{r=0}^{z-1} p(x, t, r)\right] .
\end{aligned}
$$

Now keeping $y, z$ fixed in (11), set $x=s$ and substitute $s=0,1, \cdots$, $x-1$ successively in (11) to obtain the estimate

$$
\begin{aligned}
m(x, y, z) \leqq[a(0)+b(y)+c(z)] \prod_{s=0}^{x-1} & {\left[1+\frac{\Delta a(s)}{a(s)+b(0)+c(z)}\right.} \\
& \left.+\sum_{t=0}^{y-1} \sum_{r=0}^{z-1} p(s, t, r)\right] \\
=\phi(x, y, z ; a, b, c ; p) . &
\end{aligned}
$$

Substituting this bound for $m(x, y, z)$ in (1) we obtain the desired bound in (2).

REMARK 1. We note that for the method of proof to work in Theorem 1 and all other theorems given below the following must be satisfied:

$$
\begin{aligned}
& m(x, y, z+1) \geqq m(x, y, z)>0 \quad \text { and } \quad \Delta^{2} m_{x y}(x, y, z+1) \geqq 0 ; \\
& \Delta^{2} m_{x y}(x, y, 0)=0, \quad \Delta m_{x}(x, y+1, z) \geqq 0, \quad \Delta m_{y}(x, y, z) \geqq 0 .
\end{aligned}
$$

REMARK 2. In relation to the notation $\phi$ defined in (A), we observe that Theorem 1 have hypotheses which are symmetric in 
$x, y, z$ as well as in $a, b, c$. Hence there are $3 !=6$ different conclusions we can state in Theorem 1 corresponding to the 6 permutations of $(x, y, z)$ and corresponding permutations of $(a, b, c)$. For example, in Theorem 1, we can conclude, in addition to (2) that

$$
u(x, y, z) \leqq \phi(z, x, y ; c, a, b ; p)
$$

where, by (A) above, the right side of $\left(2^{*}\right)$ is

$$
[c(0)+a(x)+b(y)] \prod_{s=0}^{z-1}\left[1+\frac{\Delta c(s)}{c(s)+a(0)+b(y)}+\sum_{t=0}^{x-1} \sum_{r=0}^{y-1} p(s, t, r)\right] .
$$

Similarly we can use $\phi(y, x, z ; b, a, c ; p)$ etc. We also note that a similar permutation applies to the conclusion of Theorem 2 given below.

Our next theorem deals with the three independent variable generalization of the discrete inequality established by Pachpatte [5, Theorem 1], which in turn is a discrete analogue of the integral inequality established by Pachpatte [9, Theorem 1].

THEOREM 2. Let $u(x, y, z), p(x, y, z)$, and $q(x, y, z)$ be real-valued nonnegative functions defined for $(x, y, z) \in N_{0}^{3}$ for which the inequality

$$
\begin{aligned}
u(x, y, z) \leqq a(a)+b(y)+c(z) & +\sum_{s=0}^{x-1} \sum_{t=0}^{y-1} \sum_{r=0}^{z-1} p(s, t, r)[u(s, t, r) \\
& \left.+\sum_{k=0}^{s-1} \sum_{l=0}^{t-1} \sum_{n=0}^{r-1} q(k, l, n) u(k, l, n)\right],
\end{aligned}
$$

holds for $(x, y, z) \in N_{0}^{3}$, where $a(x), b(y), c(z)>0, \Delta a(x), \Delta b(y), \Delta c(z) \geqq 0$, are real-valued functions defined on $N_{0}$. Then

$$
\begin{aligned}
u(x, y, z) \leqq & {[a(0)+b(y)+c(z)] } \\
& +\sum_{s=0}^{x-1}\left[\Delta a(s)+\sum_{t=0}^{y-1} \sum_{r=0}^{z-1} p(s, t, r) R(s, t, r)\right],
\end{aligned}
$$

for $(x, y, z) \in N_{0}^{3}$, where

$$
R(x, y, z)=\phi(x, y, z ; a, b, c ; p+q),
$$

for $(x, y, z) \in N_{0}^{3}$.

Proof. Define a function $m(x, y, z)$ by

$$
\begin{aligned}
& m(x, y, z)=a(x)+b(y)+c(z) \\
& \quad+\sum_{s=0}^{x-1} \sum_{t=0}^{y-1} \sum_{r=0}^{z-1} p(s, t, r)\left[u(s, t, r)+\sum_{k=0}^{s-1} \sum_{l=0}^{t-1} \sum_{n=0}^{r-1} q(k, l, n) u(k, l, n)\right],
\end{aligned}
$$

so that, by definition 


$$
\begin{aligned}
& m(0, y, z)=a(0)+b(y)+c(z), \\
& m(x, 0, z)=a(x)+b(c)+c(z), \\
& m(x, y, 0)=a(x)+b(y)+c(0) .
\end{aligned}
$$

Then by following the same steps as in the proof of Theorem 1 we have

$$
\begin{aligned}
& \Delta^{2} m_{x y}(x, y, z+1)-\Delta^{2} m_{x y}(x, y, z) \\
& =p(x, y, z)\left[u(x, y, z)+\sum_{k=0}^{x-1} \sum_{l=0}^{y-1} \sum_{n=0}^{z-1} q(k, l, n) u(k, l, n)\right]
\end{aligned}
$$

which in view of the definition of $m(x, y, z)$ implies

$$
\begin{aligned}
& \Delta^{2} m_{x y}(x, y, z+1)-\Delta^{2} m_{x y}(x, y, z) \\
& \quad \leqq p(x, y, z)\left[m(x, y, z)+\sum_{k=0}^{x-1} \sum_{l=0}^{y-1} \sum_{n=0}^{z-1} q(k, l, n) m(k, l, n)\right] .
\end{aligned}
$$

If we put

$$
v(x, y, z)=m(x, y, z)+\sum_{k=0}^{x-1} \sum_{l=0}^{y-1} \sum_{n=0}^{z-1} q(k, l, n) m(k, l, n),
$$

so that

$$
\begin{aligned}
& v(0, y, z)=a(0)+b(y)+c(z), \\
& v(x, 0, z)=a(x)+b(0)+c(z), \\
& v(x, y, 0)=a(x)+b(y)+c(0) .
\end{aligned}
$$

Then by following the same argument as in the proof of Theorem 1 we obtain

$$
\begin{aligned}
& \Delta^{2} v_{x y}(x, y, z+1)-\Delta^{2} v_{x y}(x, y, z) \\
= & \Delta^{2} m_{x y}(x, y, z+1)-\Delta^{2} m_{x y}(x, y, z)+q(x, y, z) m(x, y, z) .
\end{aligned}
$$

Using the facts that $\Delta^{2} m_{x y}(x, y, z+1)-\Delta^{2} m_{x y}(x, y, z) \leqq p(x, y, z) v(x, y, z)$ from (16) and $m(x, y, z) \leqq v(x, y, z)$ from (17) in (18) we have

$$
\Delta^{2} v_{x y}(x, y, z+1)-\Delta^{2} v_{x y}(x, y, z) \leqq[p(x, y, z)+q(x, y, z)] v(x, y, z) .
$$

Now by following the same argument as in the proof of Theorem 1 we obtain the estimate

$$
\begin{aligned}
v(x, y, z) \leqq & {[a(0)+b(y)+c(z)] \prod_{s=0}^{x-1}\left[1+\frac{\Delta a(s)}{a(s)+b(0)+c(z)}\right.} \\
& \left.+\sum_{t=0}^{y-1} \sum_{r=0}^{z-1}[p(s, t, r)+q(s, t, r)]\right]=R(x, y, z) .
\end{aligned}
$$

Substituting this bound for $v(x, y, z)$ in (16) and following the last argument as in the proof of Theorem 1, we obtain the estimate 
$m(x, y, z) \leqq[a(0)+b(y)+c(z)]+\sum_{s=0}^{x-1}\left[\Delta a(s)+\sum_{t=0}^{y-1} \sum_{r=0}^{z-1} p(s, t, r) R(s, t, r)\right]$.

Substituting this bound for $m(x, y, z)$ in (13) we obtain the desired bound in (14).

REMARK 3. We note that, if (13) holds then from the definitions of $m(x, y, z)$ and $v(x, y, z)$ we have

$$
u(x, y, z) \leqq R(x, y, z),
$$

on $N_{0}^{3}$, where $R(x, y, z)$ is defined by (15). Certainly $\left(14^{*}\right)$ is less work to compute in any given case. On the other hand, in the special case that $a, b, c$ are constant $(>0)$, and $p \equiv p_{0}, q \equiv q_{0}$ are also constants $(>0)$, then we find

$$
R(x, y, z)=(a+b+c)\left[1+\left(p_{0}+q_{0}\right) y z\right]^{x},
$$

while the bound in (14) is, say

$$
\begin{aligned}
\bar{R}(x, y, z) & =(a+b+c)\left\{1+\sum_{t=0}^{y-1} \sum_{r=0}^{z-1} \sum_{s=0}^{x-1} p_{0}\left[1+\left(p_{0}+q_{0}\right) t r\right]^{s}\right\} \\
& <(a+b+c)\left\{1+p_{0} y z \sum_{s=0}^{x-1}\left[1+\left(p_{0}+q_{0}\right) y z\right]^{s}\right\} \\
& =(a+b+c)\left\{1+\frac{p_{0}}{p_{0}+q_{0}}\left(\left[1+\left(p_{0}+q_{0}\right) y z\right]^{x}-1\right)\right\} \\
& <R(x, y, z) .
\end{aligned}
$$

Thus, in this case $\left(14^{*}\right)$ gives the simpler but not necessarily smaller bound than (14).

REMARK 4. It is interesting to note that the bounds obtained in (2) and (14) are independent of the unknown function $u(x, y, z)$. The estimates in (2) and (14) have interesting applications to uniqueness, boundedness, continuous dependence and other problems in the analysis of a class of finite difference equations involving three independent variables. Some of these applications are given in $\S 4$.

3. Furthermore inequalities. In this section we wish to establish some interesting and useful nonlinear discrete inequalities in three independent variables of the Bihari [2, pp. 8-9] and Pachpatte [4]-[8] type which can be used in the theory of finite difference equations involving three independent variables. In Theorems 3 and 4 given below we use the following notation. For $x, y, z \in N_{0}$, and functions $a, b, c$ with domain $N_{0}$, and $p$ with domain $N_{0}^{3}$, and $\Omega, V$ with domain $(0, \infty)$, set 
(B)

$$
\begin{aligned}
\Psi(x, y, z ; a, b, c ; \Omega, V(u), p)= & \Omega[a(0)+b(y)+c(z)] \\
& +\sum_{s=0}^{x-1}\left[\frac{\Delta a(s)}{V[a(s)+b(0)+c(z)]}\right. \\
& \left.+\sum_{t=0}^{y-1} \sum_{r=0}^{y-1} p(s, t, r)\right] .
\end{aligned}
$$

THEOREM 3. Let $u(x, y, z) \geqq u_{0}>0$ and $p(x, y, z) \geqq 0$ be realvalued functions defined for $(x, y, z) \in N_{0}^{3}$ and let $W$ be continuous, positive, strictly increasing function on $I=\left[u_{0}, \infty\right), u_{0}>0$. Suppose further that the inequality

$$
u(x, y, z) \leqq a(x)+b(y)+c(z)+\sum_{s=0}^{x-1} \sum_{t=0}^{y-1} \sum_{r=0}^{z-1} p(s, t, r) W(u(s, t, r)),
$$

is satisfied for $(x, y, z) \in N_{0}^{3}$, where $a(x), b(y), c(z)>0, \Delta a(x), \Delta b(y)$, $\Delta c(z) \geqq 0$, are real-valued functions defined on $N_{0}$. Then for $0 \leqq x \leqq x_{1}$, $0 \leqq y \leqq y_{1}, 0 \leqq z \leqq z_{1}$,

$$
u(x, y, z) \leqq \Omega^{-1}\{\Psi(x, y, z ; a, b, c ; \Omega, W(u), p)\},
$$

where

$$
\Omega(r)=\int_{r_{0}}^{r} \frac{d s}{W(s)}, \quad r \geqq u_{0} \quad \text { with } \quad r_{0} \geqq u_{0}
$$

$\Omega^{-1}$ is the inverse of $\Omega$ and $x_{1}, y_{1}, z_{1}$ are chosen so that

$$
\Psi(x, y, z ; a, b, c ; \Omega, W(u), p) \in \operatorname{Dom}\left(\Omega^{-1}\right),
$$

for all $x, y, z$ lying in the subintervals $0 \leqq x \leqq x_{1}, 0 \leqq y \leqq y_{1}$, $0 \leqq z \leqq z_{1}$ of $N_{0}$.

Proof. Define a function $m(x, y, z)$ by the right member of (19) so that $m(0, y, z)=a(0)+b(y)+c(z), m(x, 0, z)=a(x)+b(0)+c(z)$, $m(x, y, 0)=a(x)+b(y)+c(0)$. Then by following the same argument as in the proof of Theorem 1 we obtain

$$
\Delta^{2} m_{x y}(x, y, z+1)-\Delta^{2} m_{x y}(x, y, z)=p(x, y, z) W(u(x, y, z)),
$$

which in view of the definition of $m(x, y, z)$ and the fact that $m(x, y, z) \leqq m(x, y, z+1)$ implies

$$
\Delta^{2} m_{x y}(x, y, z+1)-\Delta^{2} m_{x y}(x, y, z) \leqq p(x, y, z) W(m(x, y, z+1)),
$$

i.e.,

$$
\frac{\Delta^{2} m_{x y}(x, y, z+1)}{W(m(x, y, z+1))}-\frac{\Delta^{2} m_{x y}(x, y, z)}{W(m(x, y, z+1))} \leqq p(x, y, z)
$$


From (22) we observe that

$$
\frac{\Delta^{2} m_{x y}(x, y, z+1)}{W(m(x, y, z+1))}-\frac{\Delta^{2} m_{x y}(x, y, z)}{W(m(x, y, z))} \leqq p(x, y, z) .
$$

Now keeping $x, y$ fixed in (23), set $z=r$ and sum over $r=0,1, \cdots$, $z-1$ to obtain the estimate

$$
\frac{\Delta^{2} m_{x y}(x, y, z)}{W(m(x, y, z))} \leqq \sum_{r=0}^{z-1} p(x, y, r) .
$$

From (24) and in view of the fact that $m(x, y, z) \leqq m(x, y+1, z)$ we observe that

$$
\frac{\Delta m_{x}(x, y+1, z)}{W(m(x, y+1, z))}-\frac{\Delta m_{x}(x, y, z)}{W(m(x, y, z))} \leqq \sum_{r=0}^{z-1} p(x, y, r)
$$

Keeping $x, z$ fixed in (25), set $y=t$ and sum over $t=0,1, \cdots, y-1$ to obtain the estimate

$$
\frac{\Delta m_{x}(x, y, z)}{W(m(x, y, z))} \leqq \frac{\Delta a(x)}{W(a(x)+b(0)+c(z))}+\sum_{t=0}^{y-1} \sum_{r=0}^{z-1} p(x, t, r) .
$$

From (21) and (26) we have

$$
\begin{aligned}
\Omega(m(x & +1, y, z))-\Omega(m(x, y, z))=\int_{m(x, y, z)}^{m(x+1, y, z)} \frac{d s}{W(s)} \\
& \leqq \frac{\Delta m_{x}(x, y, z)}{W(m(x, y, z))} \\
& \leqq \frac{\Delta a(x)}{W[a(x)+b(0)+c(z)]}+\sum_{t=0}^{y-1} \sum_{r=0}^{z-1} p(x, t, r) .
\end{aligned}
$$

Now keeping $y, z$ fixed in (27), set $x=s$ and sum over $s=0,1, \cdots$, $x-1$ to obtain the estimate

$$
\begin{aligned}
& \Omega(m(x, y, z))-\Omega(a(0)+b(y)+c(z)) \\
& \quad \leqq \sum_{s=0}^{x-1}\left[\frac{\Delta a(s)}{W[a(s)+b(0)+c(z)]}+\sum_{t=0}^{y-1} \sum_{r=0}^{z-1} p(s, t, r)\right] .
\end{aligned}
$$

The desired bound in (20) now follows by substituting the bound for $m(x, y, z)$ from (28). The subintervals of $N_{0}$ for $x, y$ and $z$ are obvious.

REMARK 5. The estimate in (20) is independent of the choice of $u_{0} \in I$ used in defining $\Omega$. One can use this fact to show that the case $u_{0} \geqq 0, W(u)>0$ on $\left(u_{0}, \infty\right)$ and $W\left(u_{0}\right)=0$ can be obtained as a limiting case from the theorem. This will allow $W(u)=u$ on $(0, \infty)$. For details, see Bessack [2, pp. 8-9]. 
REMARK 6 . If we compare Theorem 3 with $W(u) \equiv u$ for $u \geqq 1$, with Theorem 1 we see that the hypotheses (1) and (19) are then the same, but the bounds are now (2) and

$$
\begin{aligned}
u(x, y, z) \leqq[a(0)+b(y)+c(z)] & \prod_{s=0}^{x-1} \exp \left[\frac{\Delta a(s)}{a(s)+b(0)+c(z)}\right. \\
& \left.+\sum_{t=0}^{y-1} \sum_{r=0}^{z-1} p(s, t, r)\right] .
\end{aligned}
$$

Using the fact that $\exp u \geqq 1+u$ for all $u \in R$, it follows that (2) gives the better bound than $\left(20^{*}\right)$.

Our next result is a three independent variable discrete generalization of the integral inequality recently established by Pachpatte [10, Theorem 2].

THEOREM 4. Let $u(x, y, z), p(x, y, z)$ and $W$ satisfy the hypotheses of Theorem 3, and suppose further that the inequality

$$
\begin{aligned}
u(x, y, z) \leqq a(x)+b(y)+c(z) & +\sum_{s=0}^{x-1} \sum_{t=0}^{y-1} \sum_{r=0}^{z-1} p(s, t, r)[u(s, t, r) \\
& \left.+\sum_{k=0}^{s-1} \sum_{l=0}^{t-1} \sum_{n=0}^{r-1} p(k, l, n) W(u(k, l, n))\right],
\end{aligned}
$$

is satisfied for $(x, y, z) \in N_{0}^{3}$, where $a(x), b(y), c(z)>0, \Delta a(x), \Delta b(y)$, $\Delta c(z) \geqq 0$, are real-valued functions defined on $N_{0}$. Then for $0 \leqq$ $x \leqq x_{2}, 0 \leqq y \leqq y_{2}, 0 \leqq z \leqq z_{2}$,

$$
u(x, y, z) \leqq[a(0)+b(y)+c(z)]+\sum_{s=0}^{x-1}\left[\Delta a(s)+\sum_{t=0}^{y-1} \sum_{r=0}^{z-1} p(s, t, r) Q(s, t, r)\right]
$$

where

$$
Q(x, y, z)=G^{-1}\{\Psi(x, y, z ; a, b, c ; G, u+W(u), p)\},
$$

in which

$$
G(r)=\int_{r_{0}}^{r} \frac{d s}{s+W(s)}, \quad r \geqq r_{0} \quad \text { with } \quad r_{0} \geqq u_{0}
$$

$G^{-1}$ is the inverse of $G$ and $x_{2}, y_{2}, z_{2}$, are chosen so that

$$
\Psi(x, y, z ; a, b, c ; G, u+W(u), p) \in \operatorname{Dom}\left(G^{-1}\right),
$$

for all $x, y, z$ lying in the subintervals $0 \leqq x \leqq x_{2}, 0 \leqq y \leqq y_{2}$, $0 \leqq z \leqq z_{2}$ of $N_{0}$.

Proof. Define a function $m(x, y, z)$ by the right member of (29), so that $m(0, y, z)=a(0)+b(y)+c(z), m(x, 0, z)=a(x)+b(0)+c(z)$, 
$m(x, y, 0)=a(x)+b(y)+c(0)$. Then by the same argument as in the proof of Theorem 2 we obtain

$$
\begin{aligned}
\Delta^{2} m_{x y}(x, y, z+1)-\Delta^{2} m_{x y}(x, y, z) \leqq p(x, y, z) & {[m(x, y, z)} \\
& \left.+\sum_{k=0}^{x-1} \sum_{l=0}^{y-1} \sum_{n=0}^{z-1} p(k, l, n) W(m(k, l, n))\right]
\end{aligned}
$$

If we put

$$
v(x, y, z)=m(x, y, z)+\sum_{k=0}^{x-1} \sum_{l=0}^{y-1} \sum_{n=0}^{z-1} p(k, l, n) W(m(k, l, n)),
$$

so that

$$
\begin{aligned}
& v(0, y, z)=a(0)+b(y)+c(z), \\
& v(x, 0, z)=a(x)+b(0)+c(z), \\
& v(x, y, 0)=a(x)+b(y)+c(0) .
\end{aligned}
$$

Then by following the same argument as in the proof of Theorem 2 we obtain

$$
\Delta^{2} v_{x y}(x, y, z+1)-\Delta^{2} v_{x y}(x, y, z) \leqq p(x, y, z)[v(x, y, z)+W(v(x, y, z))] \cdot
$$

Now by following the same steps as in the proof of Theorem 3 we obtain the estimate

$$
\begin{aligned}
v(x, y, z) & \leqq G^{-1}[G(a(0)+b(y)+c(z)) \\
& +\sum_{s=0}^{x-1}\left[\frac{\Delta a(s)}{a(s)+b(0)+c(z)+W(a(s)+b(0)+c(z))}\right. \\
& \left.\left.+\sum_{t=0}^{y-1} \sum_{r=0}^{z-1} p(s, t, r)\right]\right]=Q(x, y, z) .
\end{aligned}
$$

Substituting this bound for $v(x, y, z)$ in (33) we have

$$
\Delta^{2} m_{x y}(x, y, z+1)-\Delta^{2} m_{x y}(x, y, z) \leqq p(x, y, z) Q(x, y, z),
$$

which implies the estimate

$$
m(x, y, z) \leqq[a(0)+b(y)+c(z)]+\sum_{s=0}^{x-1}\left[\Delta a .(s)+\sum_{t=0}^{y-1} \sum_{r=0}^{z-1} p(s, t, r) Q(s, t, r)\right] .
$$

Substituting this bound for $m(x, y, z)$ in (29) we obtain the desired bound in (30). The subintervals of $N_{0}$ for $x, y$, and $z$ are obvious.

REMARK 7. As pointed out in Remark 2 there are five other alternative conclusions corresponding to permutations of $(x, y, z)$, $(a, b, c)$, in addition to the conclusion (20) of Theorem 3. The same 
is true in case of the conclusion (30) of Theorem 4. Further we note that, if (29) holds then from the definitions of $m(x, y, z)$ and $v(x, y, z)$ we have

$$
u(x, y, z) \leqq Q(x, y, z),
$$

on $N_{0}^{3}$, where $Q(x, y, z)$ is defined by (31). In this case $\left(30^{*}\right)$ gives the simpler but not necessarily smaller bound than (30). If we compare Theorem 4 with $W(u)=u$ for $u \geqq 1$ with Theorem 2 with $p \equiv q$ we see that (13) and (29) coincide. In this case a simple analysis shows that $R(x, y, z) \leqq Q(x, y, z)$ so that the bound obtained in (14) is better than (30).

4. Some applications. In this section, we present some applications of our results to the boundedness, uniqueness, and continuous dependence of the solutions of discrete versions of hyperbolic partial differential equations involving three independent variables. It appears that these inequalities will have many applications for finite difference equations involving three independent variables, but those presented here are sufficient to convey the importance of our results.

EXAMPle 1. As a first application, we obtain a bound on the solution of a summary difference equation

$$
\Delta^{3} u_{x y z}=f\left[x, y, z, u, \sum_{s=0}^{x-1} \sum_{t=0}^{y-1} \sum_{r=0}^{z-1} h(x, y, z, s, t, r, u)\right],
$$

with given boundary conditions at $x=0, y=0, z=0$, where all the functions are defined on their respective domains of definitions and

$$
\begin{gathered}
|f[x, y, z, u, v]| \leqq p(x, y, z)[|u|+|v|], \\
|h(x, y, z, s, t, r, u)| \leqq q(s, t, r)|u|,
\end{gathered}
$$

where $p$ and $q$ satisfy the hypotheses of Theorem 2. By using the given boundary conditions, equation (35) can be represented by the equivalent summary difference equation

$$
\begin{aligned}
u(x, y, z)=g(x, y, z) & +\sum_{s=0}^{x-1} \sum_{t=0}^{y-1} \sum_{r=0}^{z-1} f[s, t, r, u(s, t, r) \\
& \left.\sum_{k=0}^{s-1} \sum_{l=0}^{t-1} \sum_{n=0}^{r-1} h(s, t, r, k, l, n, u(k, l, n))\right],
\end{aligned}
$$

where $g(x, y, z)$ depends on the given boundary conditions. If $|g(x, y, z)| \leqq a(x)+b(y)+c(z)$, where $a(x), b(y)$, and $c(z)$ are as defined in Theorem 2 , then using (36), (37) in (38) and then applying Theorem 2 , we obtain a bound on the solution $u(x, y, z)$ of (35). 
EXAMPLE 2. As a second application we establish the uniqueness of solutions of (35) with the given boundary conditions. We assume that the functions $h$ and $f$ in (35) satisfy

$$
\begin{aligned}
& |h(x, y, z, s, t, r, u)-h(x, y, z, s, t, r, \bar{u})| \leqq q(s, t, r)|u-\bar{u}|, \\
& |f[x, y, z, u, v]-f[x, y, z, \bar{u}, \bar{v}]| \leqq p(x, y, z)[|u-\bar{u}|+|v-\bar{v}|],
\end{aligned}
$$

where $p$ and $q$ are as in Example 1. The problem (35) is equivalent to the equation (38). Then for any two solutions $u$ and $\bar{u}$ of (35) we have

$$
\begin{aligned}
u-\bar{u} & =g(x, y, z)-\bar{g}(x, y, z) \\
+ & \sum_{s=0}^{x-1} \sum_{t=0}^{y-1} \sum_{r=0}^{z-1}\left\{f\left[s, t, r, u, \sum_{k=0}^{s-1} \sum_{l=0}^{t-1} \sum_{n=0}^{r-1} h(s, t, r, k, l, n, u)\right]\right. \\
- & \left.f\left[s, t, r, \bar{u}, \sum_{k=0}^{s-1} \sum_{l=0}^{t-1} \sum_{n=0}^{r-1} h(s, t, r, k, l, n, \bar{u})\right]\right\},
\end{aligned}
$$

where $g(x, y, z)$ and $\bar{g}(x, y, z)$ depends on the given boundary conditions. Using (39) and (40) in (41) and further assuming $|g-\bar{g}| \leqq \varepsilon$, for arbitrary $\varepsilon>0$, we have

$$
|u-\bar{u}| \leqq \varepsilon+\sum_{s=0}^{x-1} \sum_{t=0}^{y-1} \sum_{r=0}^{z-1} p(s, t, r)\left[|u-\bar{u}|+\sum_{k=0}^{s-1} \sum_{l=0}^{t-1} \sum_{n=0}^{r-1} q(k, l, n)|u-\bar{u}|\right] .
$$

Now a suitable application of Theorem 2 (with $a+b+c=\varepsilon$ ) gives

$$
|u(x, y, z)-\bar{u}(x, y, z)| \leqq \varepsilon+\varepsilon \sum_{s=0}^{x-1} \sum_{t=0}^{y-1} \sum_{r=0}^{z-1} p(s, t, r) K(s, t, r),
$$

where

$$
K(s, t, r)=\prod_{k=0}^{s-1}\left[1+\sum_{l=0}^{t-1} \sum_{n=0}^{r-1}(p(k, l, n)+q(k, l, n))\right],
$$

Since $\varepsilon>0$ is arbitrary we have $u=\bar{u}$, i.e., there is at most one solution of the equation (35).

We note that, here is a case where the simpler bound $|u-\bar{u}| \leqq$ $R=\varepsilon k(x, y, z)$ gives the conclusion $u \equiv \bar{u}$ more easily.

EXAMPLE 3. Our third application is an example of continuous dependence of the solution on the equation and boundary data. Consider the boundary value problem (35) given in Example 1 and

$$
\Delta^{3} U_{x y z}=F\left[x, y, z, U, \sum_{s=0}^{x-1} \sum_{t=0}^{y-1} \sum_{r=0}^{z-1} H(x, y, z, s, t, r, U)\right],
$$

with given boundary conditions at $x=0, y=0, z=0$, where all the functions are real-valued and defined on their respective domains of 
their definitions and

$$
\begin{aligned}
& \mid f\left[s, t, r, U, \sum_{k=0}^{s-1} \sum_{l=0}^{t-1} \sum_{n=0}^{r-1} H(s, t, r, k, l, n, U)\right] \\
& -F\left[s, t, r, U, \sum_{k=0}^{s-1} \sum_{l=0}^{t-1} \sum_{n=0}^{r-1} H(s, t, r, k, l, n, U)\right] \mid \leqq \varepsilon,
\end{aligned}
$$

and suppose further that the functions $h$ and $f$ in (35) satisfy the conditions (39) and (40) with $q(s, t, r)=M_{2}$ and $p(x, y, z)=M_{1}$, where $\varepsilon, M_{1}$, and $M_{2}$ are positive constants. The equations corresponding to (35) and (42) are (38) and

$$
\begin{aligned}
U(x, y, z)= & G(x, y, z)+\sum_{s=0}^{x-1} \sum_{t=0}^{y-1} \sum_{r=0}^{z-1} F[s, t, r, U(s, t, r), \\
& \left.\sum_{k=0}^{s-1} \sum_{l=0}^{t-1} \sum_{n=0}^{r-1} H(s, t, r, k, l, n, U(k, l, n))\right],
\end{aligned}
$$

where $G(x, y, z)$ depends on the given boundary conditions for the equation (42). From (38) and (44) we have

$$
\begin{aligned}
u-U=(g-G) & +\sum_{s=0}^{x-1} \sum_{t=0}^{y-1} \sum_{r=0}^{z-1}\left\{f\left[s, t, r, u, \sum_{k=0}^{s-1} \sum_{l=0}^{t-1} \sum_{n=0}^{r-1} h(s, t, r, k, l, n, u)\right]\right. \\
& \left.-F\left[s, t, r, U, \sum_{k=0}^{s-1} \sum_{l=0}^{t-1} \sum_{n=0}^{r-1} H(s, t, r, k, l, n, U)\right]\right\} .
\end{aligned}
$$

By subtracting and adding

$$
f\left[s, t, r, U, \sum_{k=0}^{s-1} \sum_{l=0}^{t-1} \sum_{n=0}^{r-1} h(s, t, r, k, l, n, U)\right]
$$

in the braces of the above equation, and further assuming $|g-G| \leqq \varepsilon$ and using (43), (39), and (40) as mentioned above we obtain

$$
|u-U| \leqq \varepsilon+\sum_{s=0}^{x-1} \sum_{t=0}^{y-1} \sum_{r=0}^{z-1}\left\{M_{1}\left[|u-U|+\sum_{k=0}^{s-1} \sum_{l=0}^{t-1} \sum_{n=0}^{r-1} M_{2}|u-U|\right]+\varepsilon\right\} .
$$

A suitable application of Theorem 2 , on the compact set $0 \leqq x, y, z \leqq C$, yields

$$
|u-U| \leqq M \varepsilon\left\{1+M_{1} \sum_{s=0}^{x-1} \sum_{t=0}^{y-1} \sum_{r=0}^{z-1} \prod_{l=0}^{s-1}\left[1+\left(M_{1}+M_{2}\right) t r\right]\right\} \leqq M^{*} \varepsilon
$$

where $M=1+C^{3}$, and $M^{*}$ is obtained by replacing $x, y, z$ by $C$ in the expression in brackets. Thus the solution of the given boundary value problem (35) depends continuously on $f$ and the boundary values. If $\varepsilon \rightarrow 0$, then $|u-U| \rightarrow 0$ on the set.

In concluding this paper we note that the inequalities and their applications presented here can be extended very easily to $n$ independent variables. We omit the details. 
ACKNOWLEDGMENT. The authors acknowledge with thanks the fruitful suggestions made by the referee for the improvement of this paper.

\section{REFERENCES}

1. E. F. Beckenbach and R. Bellman, Inequalities, Springer-Verlag, Berlin, 1961.

2. P. R. Beesack, Gronwall Inequalities, Carleton Mathematical Lecture Notes No. 11, May 1975.

3. T. H. Gronwall, Note on the derivatives with respect to a parameter of the solutions of a system of differential equations, Ann. Math., 20 (1919), 292-296.

4. B. G. Pachpatte, On the discrete generalizations of Gronwall's inequality, J. Indian Math. Soc., 37 (1973), 147-156.

5. B. G. Pachpatte, Finite difference inequalities and their applications, Proc. Nat. Acad. Sci. India, 43 (A) (1973), 348-356.

6. - On discrete inequalities related to Gronwall's inequality, Proc. Indian Acad. Sci., 85 (A) (1977), 26-40.

7. _ A note on some fundamental discrete inequalities of the Gronwall-Bellman type, Bull. Inst. Math. Acad. Sinica, 5 (1977), 121-128.

8. - On some nonlinear discrete inequalities of Gronwall type, Bull. Inst. Math. Acad. Sinica, 5 (1977), 305-315.

9. — A note on Gronwall-Bellman inequality, J. Math. Anal. Appl., 44 (1973), $758-762$.

10. An integral inequality similar to Bellman-Bihari inequality, Bull. Soc. Math. Grece, 15 (1974), 7-15.

Received April 24, 1978.

MaRATHWADA UNIVERSITY

Aurangabad., 431004

(MAHARASHTRA) INDIA 


\section{PACIFIC JOURNAL OF MATHEMATICS}

\section{EDITORS}

DoNALD BABBITT (Managing Editor)

University of California

Los Angeles, California 90024

HUGo Rossi

University of Utah

Salt Lake City, UT 84112

C. C. MOORE and ANDREW OGG

University of California

Berkeley, CA 94720
J. DUGUNDJI

Department of Mathematics University of Southern Californı Los Angeles, California 90007

R. Finn and J. Milgram Stanford University Stanford, California 94305

\section{ASSOCIATE EDITORS}

E. F. BECKENBACH

B. H. Neumann
F. WOLF

K. YOSHIDA

\section{SUPPORTING INSTITUTIONS}

UNIVERSITY OF BRITISH COLUMBIA CALIFORNIA INSTITUTE OF TECHNOLOGY UNIVERSITY OF CALIFORNIA MONTANA STATE UNIVERSITY UNIVERSITY OF NEVADA, RENO NEW MEXICO STATE UNIVERSITY OREGON STATE UNIVERSITY UNIVERSITY OF OREGON
UNIVERSITY OF SOUTHERN CALIFORNIA STANFORD UNIVERSITY UNIVERSITY OF HAWAII UNIVERSITY OF TOKYO UNIVERSITY OF UTAH WASHINGTON STATE UNIVERSITY UNIVERSITY OF WASHINGTON 


\section{Pacific Journal of Mathematics}

Vol. 82, No. $1 \quad$ January, 1979

Werner Bäni, Subspaces of positive definite inner product spaces of countable dimension ...................................... 1

Marilyn Breen, The dimension of the kernel of a planar set..............

Kenneth Alfred Byrd, Right self-injective rings whose essential right ideals

are two-sided

Patrick Cousot and Radhia Cousot, Constructive versions of Tarski's fixed

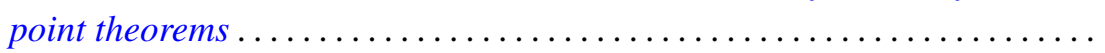

Ralph S. Freese, William A. Lampe and Walter Fuller Taylor, Congruence lattices of algebras of fixed similarity type. $I \ldots \ldots \ldots \ldots \ldots \ldots \ldots$

Cameron Gordon and Richard A. Litherland, On a theorem of Murasugi .....

Mauricio A. Gutiérrez, Concordance and homotopy. I. Fundamental

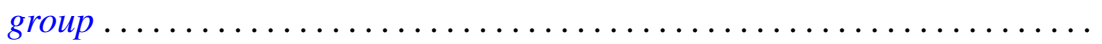

Richard I. Hartley, Metabelian representations of knot groups .............

Ted Hurley, Intersections of terms of polycentral series of free groups and free

Lie algebras ........................................

Roy Andrew Johnson, Some relationships between measures ............ 117

Oldřich Kowalski, On unitary automorphisms of solvable Lie algebras .......

Kee Yuen Lam, $K O$-equivalences and existence of nonsingular bilinear

maps...................................................

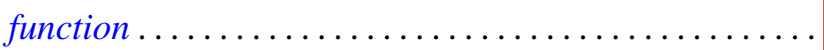

Robert A. Messer and Alden H. Wright, Embedding open 3-manifolds in compact 3-manifolds ............................

Gerald Ira Myerson, A combinatorial problem in finite fields. I . .

James Nelson, Jr. and Mohan S. Putcha, Word equations in a band of paths.

Baburao Govindrao Pachpatte and S. M. Singare, Discrete generalized Gronwall inequalities in three independent variables . .

William Lindall Paschke and Norberto Salinas, $C^{*}$-algebras associated with free products of groups ........................

Bruce Reznick, Banach spaces with polynomial norms ....

David Rusin, What is the probability that two elements of a finite group

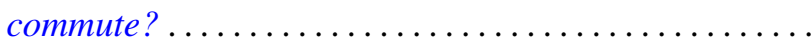

M. Shafii-Mousavi and Zbigniew Zielezny, On hypoelliptic differential operators of constant strength ...

Joseph Gail Stampfli, On selfadjoint derivation ranges .... . . .

Robert Charles Thompson, The case of equality in the matrix-valued triangle

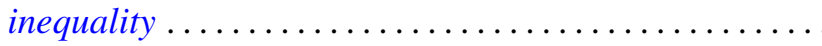

Marie Angela Vitulli, The obstruction of the formal moduli space in the negatively graded case. 